\title{
Assessment of the Influence of Demographic and Professional Characteristics on Health Care Providers' Pain Management Decisions Using Virtual Humans
}

\author{
Jeff Boissoneault, PhD; Jennifer M. Mundt, MS; Emily J. Bartley, PhD; \\ Laura D. Wandner, PhD; Adam T. Hirsh, PhD; Michael E. Robinson, PhD
}

Abstract: Disparities in health care associated with patients' gender, race, and age are well documented. Previous studies using virtual human $(\mathrm{VH})$ technology have demonstrated that provider characteristics may play an important role in pain management decisions. However, these studies have largely emphasized group differences. The aims of this study were to examine dentists' and physicians' use of VH characteristics when making clinical judgments (i.e., cue use) and to identify provider characteristics associated with the magnitude of the impact of these cues ( $\beta$-weights). Providers $(\mathrm{N}=152 ; 76$ physicians, 76 dentists) viewed video vignettes of VH patients varying in gender (male/female), race (white/black), and age (younger/older). Participants rated VH patients' pain intensity and unpleasantness and then rated their own likelihood of administering non-opioid and opioid analgesics. Compared to physicians, dentists had significantly lower $\beta$-weights associated with $\mathrm{VH}$ age cues for all ratings ( $\mathrm{p}<0.001$; $\mathrm{d}>0.69)$. These effects varied by provider race and gender. For pain intensity, professional differences were present only among non-white providers. White providers had greater $\beta$-weights than non-white providers for pain unpleasantness but only among men. Provider differences regarding the use of $\mathrm{VH}$ age cues in non-opioid analgesic administration were present among all providers except non-white males. These findings highlight the interaction of patient and provider factors in driving clinical decision making. Although profession was related to use of VH age cues in pain-related clinical judgments, this relationship was modified by providers' personal characteristics. Additional research is needed to understand what aspects of professional training or practice may account for differences between physicians and dentists and what forms of continuing education may help to mitigate the disparities.

Dr. Boissoneault is Research Assistant Professor, Department of Clinical and Health Psychology, University of Florida; Ms. Mundt is Graduate Assistant, Department of Clinical and Health Psychology, University of Florida; Dr. Bartley is Research Assistant Professor, Department of Community Dentistry and Behavioral Science, University of Florida; Dr. Wandner was Graduate Assistant, Department of Clinical and Health Psychology, University of Florida at the time of this study; Dr. Hirsh is Assistant Professor, Department of Psychology, Indiana University-Purdue University Indianapolis; and Dr. Robinson is Professor, Department of Clinical and Health Psychology, University of Florida. Direct correspondence to Dr. Michael Robinson, Department of Clinical and Health Psychology, University of Florida, P.O. Box 100165, Gainesville, FL 32610; 352-273-6153; merobin@ufl.edu.

Keywords: dentists, physicians, pain treatment, health disparities, dental education, medical education, psychosocial factors, virtual human technology

Submitted for publication 7/20/15; accepted 10/14/15

$\mathrm{H}$ ealth care providers' sensitivity to patient characteristics including gender, race, and age may underlie documented disparities in health care. For instance, women, racial/ethnic minorities, and older adults are at increased risk for undertreatment and underidentification of pain relative to their demographic counterparts. ${ }^{1-4}$ Supporting this disparity, studies utilizing virtual human (VH) technology have found that patient characteristics including gender, race, and age influence clinical decision making in health care providers. ${ }^{5-8}$ Although vignette- and retrospective chart review-based studies have traditionally been used to examine these influences, ${ }^{9,10}$ those studies often have limited ex- perimental control and ecological validity. ${ }^{11}$ The use of VH stimuli in assessing the influence of patient demographics on provider decision making allows for strict experimental control of these factors, while keeping critical factors (i.e., empirically validated pain expressions) constant between stimuli.

Providers' own demographic and professional characteristics may influence their clinical decisions directly, as well as interact with patient characteristics (i.e., cues). For instance, provider type (dentist, physician, nurse), age, gender, duration of professional experience, and minority status have been found to influence pain assessment and treatment recommendations..$^{8,11-13}$ Though informative, these 
studies have utilized nomothetic analyses emphasizing between-group differences in providers' clinical decision making. Alternatively, using idiographic approaches to examine how individual providers utilize contextual information to make clinical decisions - a lens model design ${ }^{14}$ — may provide additional insight into their decision policies.

The lens model offers a means of determining how environmental/contextual cues drive decision making processes in individuals. By systematically varying stimulus characteristics across repeated presentations and assessing the relationship between these cues and participant judgments gathered after each cue presentation, individual decision policies can be determined. ${ }^{6}$ For instance, Hirsh et al. employed a lens model-based idiographic approach to examine nurses' use of VH demographic cues (age, gender, and race) to make treatment decisions. ${ }^{15}$ Those authors found that a substantial proportion of nurses sampled gave statistically reliable weight to VH cues when judging pain intensity $(30 \%)$, pain unpleasantness (31\%), and their likelihood of administering non-opioid and opioid analgesics (13\% and $24 \%$, respectively). Interestingly, no nurses reported being aware of their biases: that their clinical decisions were influenced by VH cues. Additional work applying the lens model to both trainee and practicing physicians has identified statistically reliable genderrelated and racial biases when making treatment and referral decisions for $\mathrm{VH}$ patients. ${ }^{16-18}$ Notably, selfawareness of the use of these cues was inconsistent, with $50 \%$ of participants indicating awareness of their use of VH patient cues. ${ }^{18}$

While studies done to date have highlighted potentially critical individual biases in nurses and physicians, this idiographic approach has not, to our knowledge, been applied to dentists. Like physicians, dentists are able to prescribe opioids in every U.S. state as well as implement treatment strategies for the relief of pain or an underlying condition. Thus, identification of individuals' biases in this group, as well as the effects of provider characteristics, may help to optimize health care practice by addressing disparities and enhancing educational interventions both at the individual and group levels.

The primary aim of this study was to examine the effects of provider characteristics (gender, race, and profession) on the extent to which individual dentists and physicians weighed $\mathrm{VH}$ characteristics when making judgments of patients' pain intensity and pain unpleasantness and the practitioners' likelihood of administering non-opioid and opioid analgesics.
These weights were identified using an idiographic approach based on the lens model. ${ }^{14}$ We hypothesized that weights associated with the various $\mathrm{VH}$ cues would vary substantially among individual providers. As an empirical question, we examined the association between provider characteristics and cue weights.

\section{Materials and Methods}

All study procedures were approved by the University of Florida's Institutional Review Board (IRB \#2004-U-0503). Dentists and physicians were identified using records from Florida's Department of Business and Professional Regulation and were contacted via direct mailings advertising a web-based study on assessment and clinical decision making for pain management. Participants were included if they were currently practicing as a dentist or physician. All participants provided informed consent prior to collection of any information and were compensated $\$ 50$ for their time following study completion.

Participants completed a brief online demographic questionnaire providing information regarding age, race/ethnicity, gender, professional area, years of professional practice, and state of practice. Afterwards, they viewed 32 unique VH patient profiles consisting of a brief vignette (described below) and a looped 20-second video of a VH face. Participants were instructed to review clinical summaries fully and carefully and view each patient's video for approximately 20 seconds before providing ratings. VH faces varied systematically by gender (male/female), race (white/black), age (younger/older adult), and pain expression (low/high pain) across the 32 profiles. Empirically validated facial expressions of pain were created based on the Facial Action Coding System $^{19}$ using the PeoplePutty software package (Haptek, Inc., Santa Cruz, CA, USA) in order to differentiate between low and high pain-expressing $\mathrm{VH}$ faces. Two examples of $\mathrm{VH}$ faces are shown in Figure 1.

After viewing each profile, participants made pain assessment and treatment decision ratings using visual analogue scales (VAS) ranging from 0 to 100. Rated domains included 1) pain intensity ("no pain sensation" to "most intense pain imaginable"); 2) pain unpleasantness ("not at all unpleasant" to "most unpleasant imaginable"); 3) likelihood of administering a non-opioid analgesic ("not at all likely" to "complete certainty"); and 4) likelihood of administering an opioid analgesic ("not at all likely" 


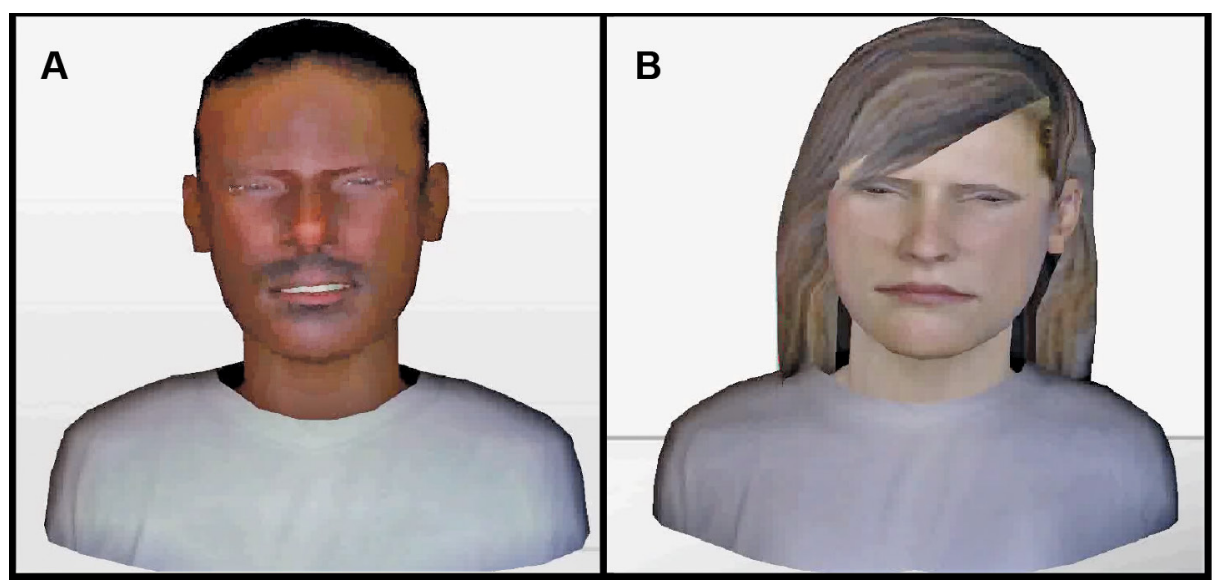

Figure 1. Examples of virtual human faces used in the study: young, black male with high pain expression (panel A) and young, white female with high pain expression (panel B)

to "complete certainty"). No time limit was imposed for viewing or rating the $\mathrm{VH}$ profiles.

The $\mathrm{VH}$ profiles included a vignette describing the patient as having low back or orofacial pain. Salience of the vignette for the two types of health care professionals included in this study was enhanced by having physicians read the vignette pertaining to low back pain and having dentists read the vignette pertaining to orofacial pain. However, both dentists and physicians viewed the same $\mathrm{VH}$ faces when making pain assessment and treatment decision ratings.

The physician vignette was as follows: "Patient presents with lower back pain for the past year of greater than one-year duration. Patient reports that the pain began after a work-related lifting incident. The pain is located in the lumbar region of the back. The pain limits patient's ability to move around freely. Patient reports no prior surgical treatments and has current prescriptions for anti-inflammatory and analgesic medications."

The dentist vignette was as follows: "The patient presents with pain in the mandibular (lower) left posterior teeth that started approximately two months ago. The patient reports having a 'large filling' placed in the tooth one year ago. Medical history is non-significant. The pain is localized to the mandibular left first molar tooth. It was initially episodic and exacerbated by cold but not warm liquids and food and was relieved somewhat by nonsteroidal anti-inflammatory drugs (NSAIDs). Approximately two weeks ago, warm food and liquids also began precipitating the pain, and it persisted after the food or liquid was removed. For the past two days, the pain has become constant, and NSAIDs fail to offer any relief."

All statistical analyses were conducted using SPSS Version 22 (SPSS, Inc., Chicago, IL, USA). After descriptive analysis of demographic variables, each participant's VAS responses to VH profiles were subjected to multiple regression analysis with $\mathrm{VH}$ characteristics (age, race, gender, and pain expression) as predictors. Outcomes of regression analysis, including standardized $\beta$-weights associated with the contribution of each VH characteristic to individual participants' VAS ratings, were recorded. $\beta$-weights provided a continuous measure of the directionality and magnitude of the impact of $\mathrm{VH}$ cues on participants' VAS ratings. For instance, a positive $\beta$-weight for $\mathrm{VH}$ age for ratings of pain intensity would indicate a provider tended to rate pain intensity higher for older than younger VHs. A Pearson's r correlation matrix was used to examine the relationship between providers' $\beta$-weights for $\mathrm{VH}$ age, gender, and race cues (across the assessment and treatment domains) and their demographic characteristics. The effects of provider characteristics on $\mathrm{VH}$ cue use were assessed using 2 (gender: male/female) X 2 (profession: dentist/physician) X 2 (race: white/non-white) ANOVA. Partial $\eta^{2}$ is reported as the effect size for F-tests, and Cohen's d is reported as the effect size for mean comparisons. The threshold for statistical significance was set at $p<0.05$. To better contextualize results and facilitate power analysis for future research, effect sizes are reported for all hypothesis tests achieving $\mathrm{p}<0.10$. 


\section{Results}

A total of 152 providers participated in the study: 76 dentists, of whom 27 were women, and 76 physicians, of whom 33 were women. Given the means by which providers were contacted, which included both emails to professional listservs and individual providers, as well as mailing hard copies of study fliers to providers' physical addresses, the precise response rate for invitations to participate in this study could not be calculated. Participants averaged 46.52 (SD 12.97) years of age and 16.99 (SD 13.60) years of professional experience, while $69 \%$ of participants self-identified as white, $11.8 \%$ as Asian, $7.2 \%$ as Hispanic, $6.6 \%$ as black/African American, and 5.3\% as another race or multiple races. For analytic purposes, providers were grouped as white $(69 \%$ of participants) and non-white $(31 \%)$. Details regarding participant demographics are shown in Table 1.

Correlations between provider characteristics and $\beta$-weights for $\mathrm{VH}$ age, gender, and race cues. Provider age did not correlate significantly with $\beta$-weights for $\mathrm{VH}$ age, gender, and race cues on any VAS ( $p>0.06)$. Years of professional experience correlated significantly with $\beta$-weights for $\mathrm{VH}$ age on pain intensity $(r=-0.22, p=0.007), \mathrm{VH}$ age on pain unpleasantness $(\mathrm{r}=-0.20, \mathrm{p}=0.01)$, and $\mathrm{VH}$ age $(\mathrm{r}=-0.18, \mathrm{p}=0.03)$ and $\mathrm{VH}$ race $(\mathrm{r}=0.24, \mathrm{p}=0.003)$ on likelihood of administering an opioid analgesic. Consequently, years of professional experience was included as a covariate in subsequent provider gender $\mathrm{X}$ profession $\mathrm{X}$ race ANOVAs (described in next paragraph). All other correlations between years of professional experience and $\beta$-weights associated with $\mathrm{VH}$ cues were non-significant ( $\mathrm{p}>0.06$ ).

Gender $X$ profession $X$ race ANOVA on $\boldsymbol{\beta}$-weights for pain intensity. A medium-to-large main effect of provider profession on the magnitude of $\beta$-weights associated with $\mathrm{VH}$ age was detected $\left(\mathrm{F}_{1,143}=14.84, \mathrm{p}<0.0001 ; \mathrm{d}=0.72\right)$, with physicians having larger $\beta$-weights than dentists. Interestingly, this effect was moderated by provider race $\left(\mathrm{F}_{1,143}=5.42, \mathrm{p}=0.02 ; \eta_{\mathrm{p}}^{2}=0.04 ;\right.$ Figure 2$)$. Differences between physicians and dentists were large among non-white providers $(\mathrm{d}=1.11)$, but small among white providers $(\mathrm{d}=0.31)$. In other words, physicians associated older $\mathrm{VH}$ age with higher pain intensity ratings. A small effect of provider race on $\beta$-weights associated with $\mathrm{VH}$ age cue (with non-white providers having higher $\beta$-weights for older age than white
Table 1. Demographic and professional characteristics of study sample $(\mathrm{N}=152)$

\begin{tabular}{|c|c|}
\hline Variable & $\begin{array}{l}\text { Mean (SD)/ } \\
\text { Percentage }\end{array}$ \\
\hline Age (years) & $46.52(12.97)$ \\
\hline Professional experience (years) & $16.99(13.60)$ \\
\hline Gender (\% women) & $39.4 \%$ \\
\hline $\begin{array}{l}\text { Race/ethnicity } \\
\text { White } \\
\text { Asian } \\
\text { Hispanic } \\
\text { Black } \\
\text { Other }\end{array}$ & $\begin{array}{c}69.0 \% \\
11.8 \% \\
7.2 \% \\
6.6 \% \\
5.3 \%\end{array}$ \\
\hline $\begin{array}{l}\text { Dental specialty }(\mathrm{n}=76) \\
\text { General dentistry } \\
\text { Operative dentistry } \\
\text { Periodontics } \\
\text { Endodontics } \\
\text { Orthodontics } \\
\text { Pediatrics } \\
\text { Other/not specified }\end{array}$ & $\begin{array}{l}68.4 \% \\
7.9 \% \\
5.3 \% \\
3.9 \% \\
3.9 \% \\
3.9 \% \\
6.7 \%\end{array}$ \\
\hline $\begin{array}{l}\text { Medical specialty }(\mathrm{n}=76) \\
\text { Internal medicine } \\
\text { Primary care } \\
\text { Surgery } \\
\text { Anesthesiology } \\
\text { Obstetrics/gynecology } \\
\text { Emergency medicine } \\
\text { Neurology } \\
\text { Psychiatry } \\
\text { Cardiology } \\
\text { Geriatrics } \\
\text { Family medicine } \\
\text { Orthopedics } \\
\text { Pediatrics } \\
\text { Other/not specified }\end{array}$ & $\begin{array}{c}25.0 \% \\
19.7 \% \\
13.2 \% \\
7.9 \% \\
6.6 \% \\
3.9 \% \\
3.9 \% \\
2.6 \% \\
1.3 \% \\
1.3 \% \\
1.3 \% \\
1.3 \% \\
1.3 \% \\
10.7 \%\end{array}$ \\
\hline
\end{tabular}

providers) approached but did not reach significance $\left(\mathrm{F}_{1,143}=3.23, \mathrm{p}=0.07 ; \mathrm{d}=0.32\right)$. No other provider characteristics were found to influence the weighing of VH cues when making judgments regarding pain intensity. Detailed information regarding the outcomes of these analyses are summarized in Table 2 and visualized in Figure 3.

Gender $X$ profession $X$ race ANOVA on $\boldsymbol{\beta}$-weights for pain unpleasantness. As for pain intensity, analyses indicated physicians weighed $\mathrm{VH}$ age more heavily when making pain unpleasantness ratings than did dentists $\left(\mathrm{F}_{1,143}=20.68, \mathrm{p}<0.0001\right.$; $\mathrm{d}=0.85$ ). An interactive effect of provider gender and race was also identified $\left(\mathrm{F}_{1,143}=4.60, \mathrm{p}=0.03\right.$; 


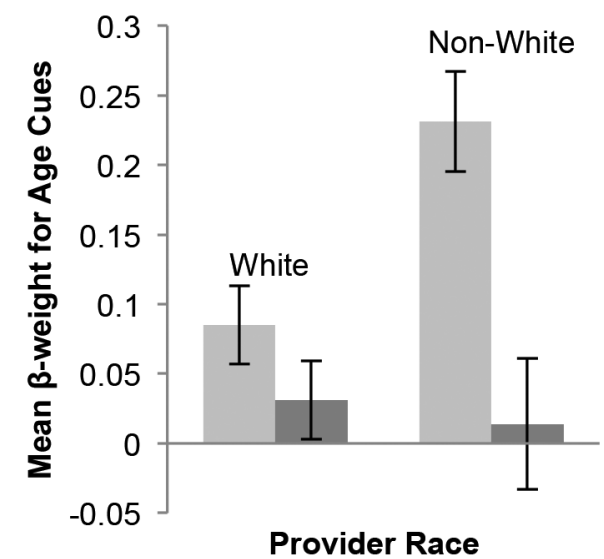

Physicians Dentists

Figure 2. Use of age cues for estimating pain intensity: race $X$ profession interaction

Note: Error bars represent standard errors.

$\left.\eta_{\mathrm{p}}^{2}=0.03\right)$ such that white providers had higher $\beta$-weights for female VHs than non-white providers, but only among men $(\mathrm{d}=0.70)$. No other provider characteristics were found to influence the weighting of $\mathrm{VH}$ cues when making judgments regarding pain unpleasantness (Table 2, Figure 3).

Gender $X$ profession $X$ race ANOVA on $\beta$-weights for non-opioid analgesic administration. Characterization of an interaction among provider gender, race, and profession $\left(\mathrm{F}_{1,140}=4.34, \mathrm{p}=0.04\right.$; $\left.\eta_{\mathrm{p}}^{2}=0.03\right)$ revealed that white male, white female, and non-white female physicians weighed $\mathrm{VH}$ age more heavily than did their dental counterparts. Effect size ranged between medium-to-large for white men and women $(\mathrm{d}=0.69)$ and large for non-white women $(\mathrm{d}=1.25)$. However, no difference between professions was noted for non-white men.

Analyses also revealed small, statistically unreliable effects of provider gender on weight given to $\mathrm{VH}$ gender $\left(\mathrm{F}_{1,140}=3.30, \mathrm{p}=0.07 ; \mathrm{d}=0.35\right)$ and age $\left(\mathrm{F}_{1,140}=3.22, \mathrm{p}=0.08 ; \mathrm{d}=0.32\right)$ when making judgments regarding likelihood of administering a non-opioid analgesic, with female providers giving greater weight to female $\mathrm{VH}$ gender than male providers. A statistically small main effect of provider race suggesting non-white providers weighed $\mathrm{VH}$ race (i.e., minority status) more heavily than white providers also approached significance $\left(\mathrm{F}_{1,140}=3.37\right.$, $\mathrm{p}=0.07 ; \mathrm{d}=0.37$ ). No other provider characteristics were found to influence the weighting of $\mathrm{VH}$ cues when making judgments regarding administration of non-opioid analgesics (Table 3, Figure 3).

Gender $X$ profession $X$ race ANOVA on $\beta$-weights for opioid analgesic administration. A medium-to-large effect of provider profession suggested that, similar to the other decision domains, physicians weighed $\mathrm{VH}$ age more heavily when making judgments regarding opioid administration $\left(\mathrm{F}_{1,142}=13.76, \mathrm{p}<0.0001 ; \mathrm{d}=0.70\right)$. However, no effects of provider characteristics on the use of $\mathrm{VH}$ cues were detected (Table 3, Figure 3).

\section{Discussion}

Previous studies have demonstrated that patient characteristics such as gender, race, and age influence health care provider decisions in pain assessment and treatment, ${ }^{1-4,20,21}$ an effect that may underlie pain management disparities. Additionally, there is evidence that providers' own characteristics impact their pain assessment and treatment decisions. ${ }^{8,11-13}$ Using an idiographic analytic approach based on the lens model,,${ }^{14}$ this study extends previous work by examining the extent to which provider characteristics predicted the weight that individual providers ascribed to $\mathrm{VH}$ patient demographic cues (gender, race, age) when assessing and treating pain.

We identified a consistent main effect of profession such that physicians weighted age cues of the $\mathrm{VH}$ patient more strongly than dentists for every rating scale. More specifically, positive $\beta$-weights for physicians indicated that older age was associated with higher ratings for pain intensity, pain unpleasantness, likelihood of administering a nonopioid analgesic, and likelihood of administering an opioid analgesic. Interestingly, physicians' use of age cues was not universally higher than dentists for ratings of pain intensity and non-opioid administration, suggesting the presence of subgroups for which the general pattern of higher age cue use by physicians might not hold. Specifically, this pattern was not maintained in white providers' estimates of pain intensity and in non-white males' likelihood of administering non-opioid analgesics. The racial and ethnic heterogeneity of the non-white providers precludes a definitive interpretation of these interactions. However, in a broad sense, these results suggest that the use of age cues for pain management-related decisions is related to factors beyond professional 


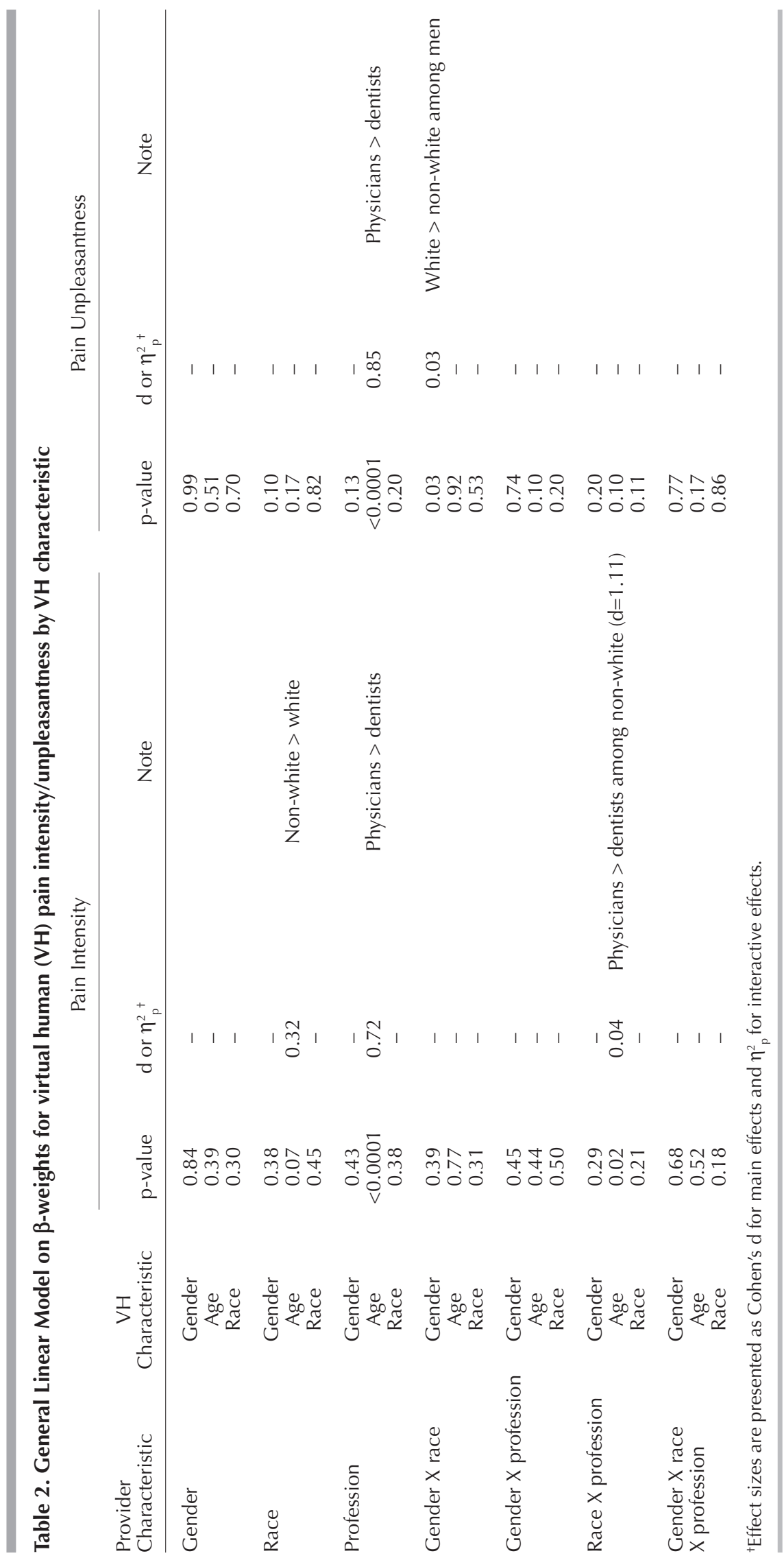




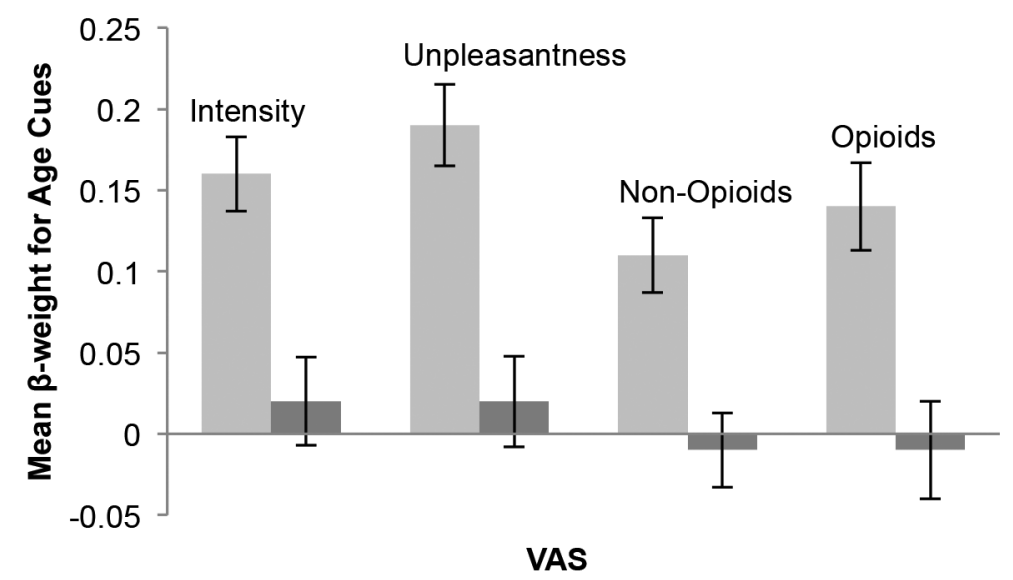

Physicians Dentists

Figure 3. Use of age cues by physicians and dentists

Note: Error bars represent standard errors.

training and may be influenced by personal or cultural factors such as gender and race or by selection factors determining choice of profession.

Nonetheless, the overall pattern of age cue use varying by profession raises interesting questions about the influence of professional training and practice settings on clinical decision making related to pain management. Indeed, similar to previous (non-idiographic) analyses of professional effects on $\mathrm{VH}$ cue use in pain-related clinical decision making, ${ }^{12}$ main effects of profession were quite robust across measures. The mechanism underlying this effect is unclear. Dental and medical students receive relatively little formal coursework specific to pain, although pain is one of the top reasons patients seek care. ${ }^{22,23}$ However, it is possible that, in the course of their training, dentists and physicians receive differing instruction on the assessment and treatment of pain. Further, the use of specific demographic cues may be predicated on the differences in patient characteristics observed across these provider settings. For instance, dentists treat a relatively limited range of pain conditions compared to physicians, who are tasked with treating all types of bodily pain as well as accompanying medical comorbidities. When conceptualizing a complex pain presentation, resorting to relatively simple heuristics (i.e., demographic cues such as age) may assist physicians in efficiently making decisions regarding their patients' treatment. In a related manner, differences in time constraints may also explain differences in physicians' and dentists' use of demographic cues. In fact, recent surveys of U.S. providers found that physicians see roughly twice as many patients per week as dentists: as of 2009 , dentists in private practice reported seeing 53.2 patients per week, ${ }^{24}$ while a 2008 survey of physicians indicated they saw an average of 117.2 patients per week. ${ }^{25}$ Hence, heuristics may be more influential when rapid decision making is needed. ${ }^{26}$

\section{Strengths and Limitations}

This study had a number of methodological strengths. First, the validity of these results for dentists and physicians is enhanced by the use of patient vignettes tailored to each profession (tooth pain for dentists and low back pain for physicians) in order to capture differences in typical patient presentations seen in medical and dental settings. Because physicians rarely treat orofacial pain (excluding, of course, a neurologist treating a patient with trigeminal neuralgia) and dentists do not typically treat musculoskeletal pain other than in the face, professionally realistic vignettes should improve ecological validity of participants' ratings. Second, the use of standardized $\mathrm{VH}$ patients with empirically validated pain expressions allowed for a degree of experimental control and ecological validity not present in many previous studies on this subject. The use of reliable 


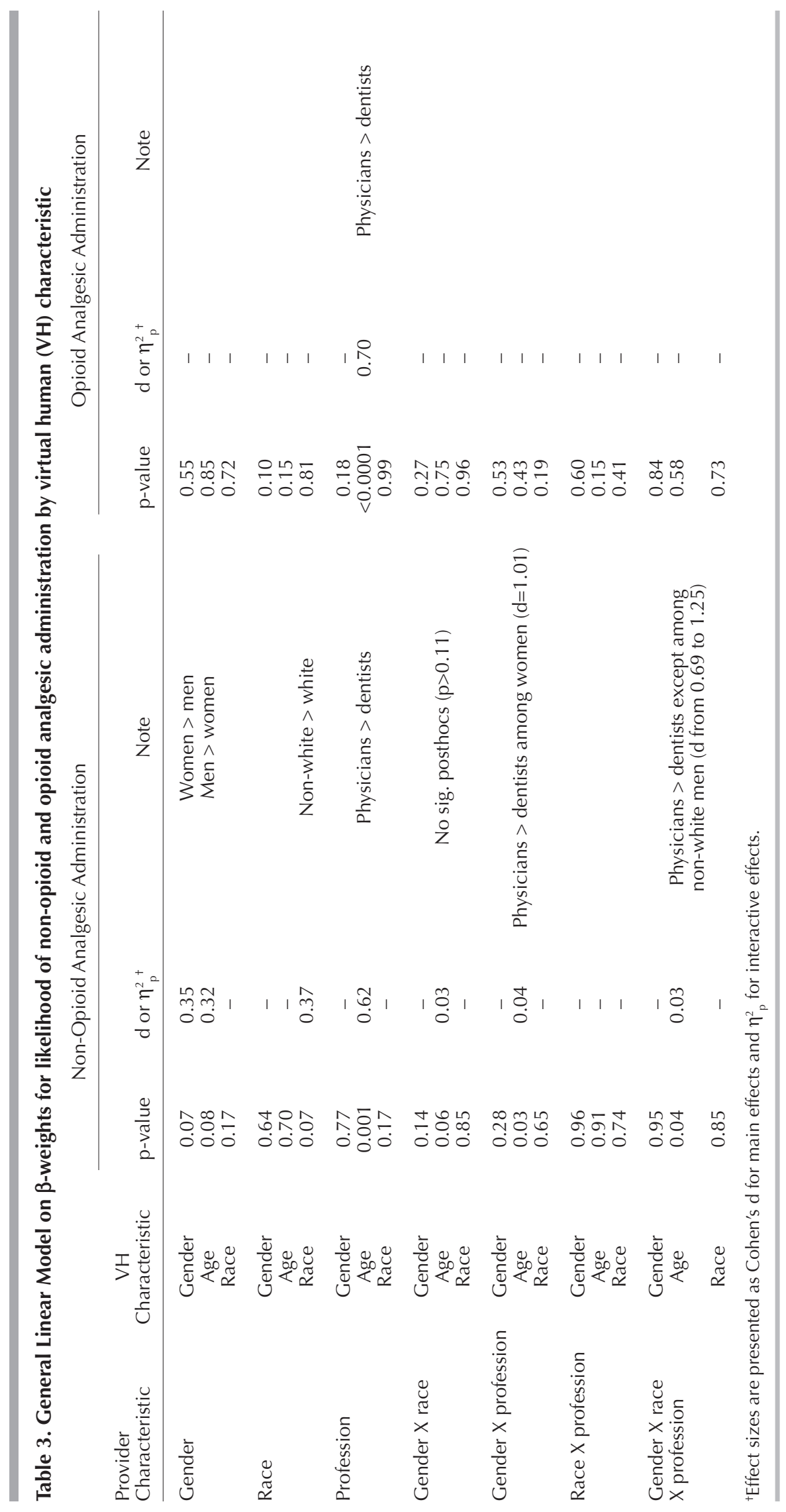


and valid rating scales represents an additional methodological strength.

Although there were several strengths in the study, some limitations merit discussion. First, the patterns observed may not be generalized to provider behavior in clinical settings. Second, the study sample included a proportion of minority providers similar to that of the U.S. population as a whole, ${ }^{27}$ therefore, the relatively small size of our sample required dichotomous labeling of race as simply white or non-white. In future studies, a larger sample size and explicit oversampling of minorities would allow for examination of differences between providers of different racial and ethnic minority groups. The VH patients were similarly of only two racial categories (white and black), and future research should examine whether providers' ratings vary across $\mathrm{VH}$ patients of different racial and ethnic groups.

Finally, the vignettes used in this study differed with regard to the time frame for pain onset: two months with recent exacerbation for the dentistry vignette and one year for the physician vignette. This discrepancy may represent a confounding factor in comparisons of the weighting of virtual human cues between professions, particularly with regard to judgments regarding analgesic administration because it is well known that opioid medications are less effective for chronic than acute or sub-acute pain. ${ }^{28}$ However, we believe it is unlikely that the weighting of virtual human age for judgments of pain intensity and unpleasantness would be confounded by this difference in time frame between vignettes. It is also unclear how a difference in pain chronicity between vignettes would drive detected differences in the use of virtual human age cues between professions. Regardless, future studies should use vignettes that do not vary in degree of pain chronicity at clinical presentation in order to avoid this potential confounding factor.

\section{Clinical Implications and Future Research}

Although other health professionals routinely treat individuals with chronic pain, only dentists and physicians are able to prescribe opioid analgesics in all states. The results of our study therefore have important clinical implications, given the critical role of these professionals in pain management. This study demonstrated that patient and provider characteristics interact to influence pain assessment and prescription behavior. Notably, profession type and personal characteristics of providers influenced the weight given to various demographic characteristics when assessing virtual patients' pain. This suggests that both training and personal experiences come to bear when making pain-related clinical judgments. Only a small number of patient and provider characteristics were considered in this study, and it is likely that actual clinical encounters are shaped by the interaction of numerous other factors related to patient and provider culture, behavior, expectations, and physical characteristics. Future research should examine additional patient characteristics such as socioeconomic status or attractiveness.

Differences in professional training, practice setting, or specialty may also be important for understanding why physicians and dentists showed differences in $\mathrm{VH}$ cue use, and these variables should be considered in future research. In addition, although physicians were found to weigh $\mathrm{VH}$ age more heavily than dentists when making pain-related clinical judgments, it is unclear whether this bias corresponds to over- or undertreatment of older adults' pain. This question should be explicitly examined in future studies.

$\mathrm{VH}$ technology offers a potential vehicle for providing educational interventions to address provider biases. The idiographic approach described in this study may be utilized to provide personalized feedback to providers, thus increasing awareness of their tendencies to use certain demographic cues when making clinical decisions. Taking this a step further, normative feedback would provide insight into how their cue-use tendencies differ from those of their peers. Furthermore, interactions with VH patients could be used in order to modify provider perceptions and thereby reduce decision making biases. Although this study focused on pain-related clinical judgments, these principles could be applied to the assessment and treatment of a wide variety of clinical scenarios. Ultimately, the efficacy of such interventions should be measured in terms of outcomes with actual patients.

\section{Conclusion}

While previous research has demonstrated disparities in chronic pain care based on patient demographic characteristics, the results of this study highlight the interaction of patient and provider factors in driving pain-related clinical judgments. Overall, our findings indicate that profession may be related to age cue use, but this relationship was 
modified by personal characteristics (race, gender) of providers. More research is needed to understand what aspects of professional training or practice setting may account for differences between physicians and dentists and what forms of continuing education may help to mitigate the disparities. Future research on pain-related clinical decision making using $\mathrm{VH}$ patients has the potential to identify other influential aspects of the patient-provider interaction, assess provider biases, and offer an experiential intervention that may modify those biases.

\section{Acknowledgments}

This study was supported in part by grants from the National Institute of Dental and Craniofacial Research (R01DE013208) to Dr. Michael E. Robinson. Dr. Jeff Boissoneault was supported by an NINDS training grant T32NS045551 to the University of Florida Pain Research and Intervention Center of Excellence.

\section{Disclosure}

None of the authors reported any conflicts of interest.

\section{REFERENCES}

1. Tamayo-Sarver JH, Dawson NV, Hinze SW, et al. The effect of race/ethnicity and desirable social characteristics on physicians' decisions to prescribe opioid analgesics. Acad Emerg Med 2003;10(11):1239-48.

2. Olsen Y, Daumit GL, Ford DE. Opioid prescriptions by U.S. primary care physicians from 1992 to 2001. J Pain 2006;7(4):225-35.

3. Chen EH, Shofer FS, Dean AJ, et al. Gender disparity in analgesic treatment of emergency department patients with acute abdominal pain. Acad Emerg Med 2008;15(5):414-8.

4. Anderson KO, Mendoza TR, Valero V, et al. Minority cancer patients and their providers: pain management attitudes and practice. Cancer 2000;88(8):1929-38.

5. Alqudah AF, Hirsh AT, Stutts LA, et al. Sex and race differences in rating others' pain, pain-related negative mood, pain coping, and recommending medical help. J Cyber Ther Rehabil 2010;3(1):63-70.

6. Hirsh AT, George SZ, Robinson ME. Pain assessment and treatment disparities: a virtual human technology investigation. Pain 2009;143(1-2):106-13.

7. Hirsh AT, Callander SB, Robinson ME. Patient demographic characteristics and facial expressions influence nurses' assessment of mood in the context of pain: a virtual human and lens model investigation. Int J Nurs Stud 2011;48(11):1330-8.

8. Wandner LD, Hirsh AT, Torres CA, et al. Using virtual human technology to capture dentists' decision policies about pain. J Dent Res 2013;92(4):301-5.
9. Nampiaparampil DE, Nampiaparampil JX, Harden RN. Pain and prejudice. Pain Med 2009;10(4):716-21.

10. Weisse CS, Sorum PC, Dominguez RE. The influence of gender and race on physicians' pain management decisions. J Pain 2003;4(9):505-10.

11. Bartley EJ, Boissoneault J, Vargovich AM, et al. The influence of health care professional characteristics on pain management decisions. Pain Med 2015;16(1):99-111.

12. Wandner LD, Heft MW, Lok BC, et al. Healthcare professionals' pain assessment and treatment decisions using virtual human technology. Presentation at International Association for the Study of Pain, 14th World Congress on Pain, Milan, Italy, 2012.

13. Wandner LD, Heft MW, Lok BC, et al. The impact of patients' gender, race, and age on health care professionals' pain management decisions: an online survey using virtual human technology. Int J Nurs Stud 2014;51(5):726-33.

14. Stewart TR. The lens model. In: Kattan M, ed. Encyclopedia of medical decision making. Thousand Oaks, CA: Sage, 2009.

15. Hirsh AT, Jensen MP, Robinson ME. Evaluation of nurses' self-insight into their pain assessment and treatment decisions. J Pain 2010;11(5):454-61.

16. Hirsh AT, Hollingshead NA, Ashburn-Nardo L, et al. The interaction of patient race, provider bias, and clinical ambiguity on pain management decisions. J Pain 2015;16(6):558-68.

17. Hirsh AT, Hollingshead NA, Bair MJ, et al. The influence of patient's sex, race, and depression on clinician pain treatment decisions. Eur J Pain 2013;17(10):1569-79.

18. Hollingshead NA, Matthias MS, Bair MJ, et al. Impact of race and sex on pain management by medical trainees: a mixed methods pilot study of decision making and awareness of influence. Pain Med 2015;16(2):280-90.

19. Ekman P, Friesen W, Hager J. Facial action coding system. Salt Lake City: A Human Face, 2002.

20. Denny DL, Guido GW. Undertreatment of pain in older adults: an application of beneficence. Nurs Ethics 2012;19(6):800-9.

21. Hadjistavropoulos T, Herr K, Turk DC, et al. An interdisciplinary expert consensus statement on assessment of pain in older persons. Clin J Pain 2007;23(1 Suppl):S1-43.

22. Pinto A, Khalaf M, Miller CS. The practice of oral medicine in the United States in the twenty-first century: an update. Oral Surg Oral Med Oral Pathol Oral Radiol 2015;119(4):408-15.

23. Gaskin DJ, Richard P. The economic costs of pain in the United States. J Pain 2012;13(8):715-24.

24. American Dental Association. 2010 survey of dental practice: characteristics of dentists in private practice and their patients. Chicago: American Dental Association, 2012.

25. A survey of America's physicians: practice patterns and perspectives. Boston: The Physicians Foundation, 2012.

26. Klein JG. Five pitfalls in decisions about diagnosis and prescribing. BMJ 2005;330(7494):781-3.

27. Humes KR, Jones NA, Ramirez RR, et al. Overview of race and Hispanic origin: 2010. Washington, DC: U.S. Department of Commerce, Economics and Statistics Administration, U.S. Census Bureau, 2011.

28. Manchikanti L, Benyamin R, Datta S, et al. Opioids in chronic noncancer pain. Expert Rev Neurother 2010; 10:775-89. 